\title{
A FUNCTIONAL ANALYSIS POINT OF VIEW ON ARZELA-ASCOLI THEOREM
}

\author{
GABRIEL NAGY
}

\begin{abstract}
We discuss the Arzela-Ascoli pre-compactness Theorem from the point of view of Functional Analysis, using compactness in $\ell^{\infty}$ and its dual.
\end{abstract}

The Arzela-Ascoli Theorem is a very important technical result, used in many branches of mathematics. Aside from its numerous applications to Partial Differential Equations, the Arzela-Ascoli Theorem is also used as a tool in obtaining Functional Analysis results, such as the compactness for duals of compact operators, as presented for example [1]. The purpose of this note is to offer a new perspective on the Arzela-Ascoli Theorem based on a functional analytic proof.

The theorem of Arzela and Ascoli deals with (relative) compactness in the Banach space $C(K)$ of complex valued continuous functions on a compact Hausdorff space $K$. One helpful characterization of pre-compactness for sets in a complete metric space is the following well-known criterion, which we state without proof.

Proposition 1. Let $(\mathcal{Y}, d)$ be a complete metric space. For a subset $\mathcal{M} \subset \mathcal{Y}$, the following are equivalent:

(i) $\mathcal{M}$ is relatively compact in $\mathcal{Y}$, i.e. its closure $\overline{\mathcal{M}}$ in $\mathcal{Y}$ is compact;

(ii) $\mathcal{M}$ contains no infinite subsets $\mathcal{T}$, satisfying

$$
\inf \{d(x, y): x, y \in \mathcal{T}, x \neq y\}>0 .
$$

Proposition 2. Let $\mathcal{X}$ be a normed vector space, let $\mathcal{S} \subset \mathcal{X}$ be a compact subset, and let $\mathcal{B}$ be the unit ball in $\mathcal{X}^{*}$ - the (topological) dual of $\mathcal{X}$ - equipped with the $w^{*}$ topology. If we consider the Banach algebra $\mathcal{A}=C(\mathcal{S})$, equipped with the uniform topology, then the restriction map $\Theta:\left.\left(\mathcal{B}, w^{*}\right) \ni \phi \longmapsto \phi\right|_{\mathcal{S}} \in(\mathcal{A},\|\cdot\|)$ is continuous. In particular, the set $\Theta(\mathcal{B})$ is compact in $\mathcal{A}$.

Proof. To prove contnuity, we start with a net $\left(\phi_{\lambda}\right)_{\lambda \in \Lambda}$ in $\mathcal{B}$, that converges to some $\phi \in \mathcal{B}$ in the $w^{*}$-topology, and let us show that the net $\left(\left.\phi_{\lambda}\right|_{\mathcal{S}}\right)_{\lambda \in \Lambda}$ converges to $\left.\phi\right|_{\mathcal{S}}$ uniformly on $\mathcal{S}$. Fix some $\varepsilon>0$, and (use compactness of $\mathcal{S}$ ) choose points $s_{1}, \ldots, s_{n} \in \mathcal{S}$, such that

$(*)$ for every $s \in \mathcal{S}$, there exists $k \in\{1, \ldots, n\}$, with $\left\|s-s_{k}\right\|<\varepsilon / 3$.

Using the condition $\phi_{\lambda} \stackrel{w^{*}}{\longrightarrow} \phi$, there exists $\lambda_{\varepsilon} \in \Lambda$, such that

$$
\left|\phi_{\lambda}\left(s_{k}\right)-\phi\left(s_{k}\right)\right|<\varepsilon / 3, \quad \forall \lambda \geq \lambda_{\varepsilon}, k \in\{1, \ldots, n\} .
$$

Now we are done, since if we start with some arbitrary $s \in \mathcal{S}$, and we choose $k \in\{1, \ldots, n\}$, such that $\left\|s-s_{k}\right\|<\varepsilon / 3$, then using (1) we get

$$
\begin{aligned}
\left|\phi_{\lambda}(s)-\phi(s)\right| & \leq\left|\phi_{\lambda}(s)-\phi_{\lambda}\left(s_{k}\right)\right|+\left|\phi_{\lambda}\left(s_{k}\right)-\phi\left(s_{k}\right)\right|+\left|\phi\left(s_{k}\right)-\phi(s)\right| \leq \\
& \leq\left\|\phi_{\lambda}\right\| \cdot\left\|s-s_{k}\right\|+\left|\phi_{\lambda}\left(s_{k}\right)-\phi\left(s_{k}\right)\right|+\|\phi\| \cdot\left\|s-s_{k}\right\| \leq \\
& \leq 2\left\|s-s_{k}\right\|+\left|\phi_{\lambda}\left(s_{k}\right)-\phi\left(s_{k}\right)\right| \leq \varepsilon, \quad \forall \lambda \geq \lambda_{\varepsilon} .
\end{aligned}
$$


Having proven the continuity of $\Theta$, the second assertion follows from Alaoglu's Theorem (which states that $\mathcal{B}$ is compact in the $w^{*}$-topology).

Theorem (Arzela-Ascoli). Let $K$ be a compact Hausdorff space, and let $\mathcal{M} \subset C(K)$ be a set which is

- pointwise bounded: $\sup \{|f(p)|: f \in \mathcal{M}\}<\infty, \forall p \in K$;

- equicontinuous: for every $p \in K$ and $\varepsilon>0$, there exists a neighborhood $N_{p, \varepsilon}$ of $p$ in $K$, such that: $\sup _{f \in \mathcal{M}}|f(q)-f(p)| \leq \varepsilon, \forall q \in N_{p, \varepsilon}$.

Then $\mathcal{M}$ is relatively compact in $C(K)$ in the uniform topology.

Proof. The main observation is that, using pointwise boundedness, any set $\mathcal{T} \subset \mathcal{M}$ gives rise to a map

$$
\Phi_{\mathcal{T}}: K \ni p \longmapsto[f(p)]_{f \in \mathcal{T}} \in \ell^{\infty}(\mathcal{T}) .
$$

(Here $\ell^{\infty}(\mathcal{T})$ denotes the Banach space of all bounded functions from $\mathcal{T}$ to $\mathbb{C}$.), Furthermore, by equicontinuity the map $\Phi_{\mathcal{T}}$ is in fact continuous, when $\ell^{\infty}(\mathcal{T})$ is equipped with the norm topology. Since $K$ is compact, so is the set

$$
\mathcal{S}_{\mathcal{T}}=\Phi_{\mathcal{T}}(K) \subset \ell^{\infty}(\mathcal{T}) .
$$

We are going to argue by contradiction (see Proposition 1), assuming the existence of some $\rho>0$, and of an infinite set $\mathcal{T} \subset \mathcal{M}$, such that

$$
\|f-g\|>\rho, \forall f, g \in \mathcal{T}, f \neq g .
$$

Consider now the unit ball $\mathcal{B}$ in the dual space $\ell^{\infty}(\mathcal{T})^{*}$, and use Proposition 2 to conclude that the set

$$
\Theta_{\mathcal{T}}=\left\{\left.\phi\right|_{\mathcal{S}_{\mathcal{T}}}: \phi \in \mathcal{B}\right\} \subset C\left(\mathcal{S}_{\mathcal{T}}\right)
$$

is compact in $C\left(\mathcal{S}_{\mathcal{T}}\right)$ in the norm topology. Consider now the coordinate maps $e_{f}: \ell^{\infty}(\mathcal{T}) \rightarrow \mathbb{C}, f \in \mathcal{T}$, and their restrictions $\theta_{f}=\left.e_{f}\right|_{\mathcal{S}_{\mathcal{T}}} \in C\left(\mathcal{S}_{\mathcal{T}}\right)$, which satisfy

$$
\theta_{f}\left(\Phi_{\mathcal{T}}(p)\right)=f(p), \quad \forall p \in K, f \in \mathcal{T} .
$$

Now, if we start with $f, g \in \mathcal{T}, f \neq g$, then using (2) there exists $p \in K$, such that $|f(p)-g(p)|>\rho$, so by (3) we also get

$$
\left|\theta_{f}\left(\Phi_{\mathcal{T}}(p)\right)-\theta_{g}\left(\Phi_{\mathcal{T}}(p)\right)\right|>\rho .
$$

This way we have shown that

$$
\left\|\theta_{f}-\theta_{g}\right\|_{C\left(\mathcal{S}_{\mathcal{T}}\right)}>\rho, \quad \forall f, g \in \mathcal{T}, f \neq g
$$

and therefore the set $\Theta_{\mathcal{T}}$ - which contains all the $\theta_{f}$ 's - cannot be compact in the norm topology.

\section{REFERENCES}

[1] W. Rudin, Functional Analysis, McGraw-Hill, Springer-Verlag, New York, 1973

Department of Mathematics, Kansas State university, Manhattan KS 66506, U.S.A.

E-mail address: nagy@math.ksu.edu 